\title{
Exact density matrix for a two-electron model atom and approximate proposals for realistic two-electron systems
}

\author{
C. Amovilli ${ }^{1}$ and N. H. March ${ }^{2,3}$ \\ ${ }^{1}$ Dipartimento di Chimica e Chimica Industriale, Università di Pisa, Via Risorgimento 35, 56126 Pisa, Italy \\ ${ }^{2}$ Department of Physics, University of Antwerp (RUCA), Groenenborgerlaan 171, B-2020 Antwerp, Belgium \\ ${ }^{3}$ Oxford University, Oxford, England \\ (Received 20 June 2002; published 28 February 2003)
}

\begin{abstract}
Moshinsky introduced an exactly soluble model of a two-electron atom consisting of two spin- $\frac{1}{2}$ particles interacting via harmonic forces and moving in a harmonic-oscillator potential. Here, the exact ground-state density $\rho(r)$ is related to the (also analytically known) Hartree-Fock density $\rho_{H F}(r)$. The generalization to the off-diagonal matrix $\gamma\left(\mathbf{r}, \mathbf{r}^{\prime}\right)$ is then effected, this being related to the idempotent $\gamma_{H F}\left(\mathbf{r}, \mathbf{r}^{\prime}\right) / 2$. This exact information on this "model atom" prompts us to propose an approximate form of $\gamma\left(\mathbf{r}, \mathbf{r}^{\prime}\right)$ for the He-like ions, the $\mathrm{H}_{2}$ molecule and, in general, all two-electron systems. $\gamma\left(\mathbf{r}, \mathbf{r}^{\prime}\right)$ is constructed solely from the exact $\rho(\mathbf{r})$ and its Hartree-Fock counterpart. Some detailed treatment of the two-electron Hookean atom with spring constant $k=1 / 4$ (atomic units) is also presented.
\end{abstract}

DOI: 10.1103/PhysRevA.67.022509

PACS number(s): 31.15.Ew, 31.25.Eb, 31.25.Nj

\section{INTRODUCTION}

A good deal of interest is now focussing on the low-order density matrices in the description of the ground-state properties of finite systems such as atoms, molecules, and clusters. One reason for this is the proof by Holas and March [1] that the exact exchange-correlation potential $V_{x c}(\mathbf{r})$ of the density-functional theory [2] can be written explicitly in terms of the one-particle density matrix $\gamma\left(\mathbf{r}, \mathbf{r}^{\prime}\right)$ and the diagonal element $n_{2}\left(\mathbf{r}_{1}, \mathbf{r}_{2}\right)$ of the two-particle density matrix (see also Ref. [3]). Second, the proposal of Goedecker and Umrigar [4], while not without some difficulties, is already recognized as a successful step forward in developing a density-matrix-functional approach to the ground-state problem of a correlated electronic assembly [5].

The present proposal lies in this same general area. However, we have had in mind the essential philosophy underlying the Mфller-Plesset perturbation theory, namely to construct a treatment that has the Hartree-Fock (HF) solution as its zero-order term. Of course, for two-electron systems such as He-like atomic ions with atomic number $Z$, or the $\mathrm{H}_{2}$ molecule, the HF one-particle density matrix (1DM) has the form

$$
\gamma_{H F}\left(\mathbf{r}, \mathbf{r}^{\prime}\right)=\rho_{H F}^{1 / 2}(\mathbf{r}) \rho_{H F}^{1 / 2}\left(\mathbf{r}^{\prime}\right) .
$$

The outline of the paper is as follows. In Sec. II the harmonic model of Moshinsky is set out and applied to obtain the correlation kinetic energy. This then motivates a proposal for the correlated one-particle density matrix $\gamma\left(\mathbf{r}, \mathbf{r}^{\prime}\right)$. The approximation employed in constructing $\gamma\left(\mathbf{r}, \mathbf{r}^{\prime}\right)$ is then tested in Sec. III on the two-electron Hookean atom. Sections IV and $\mathrm{V}$ present results for He-like atomic ions and for the $\mathrm{H}_{2}$ molecule, respectively, while Sec. VI constitutes a summary and proposals for possible future studies.

\section{THE MOSHINSKY MODEL}

Before turning to discuss the "real" two-electron systems, inevitably by approximate methods, let us begin with the soluble model of Moshinsky [6]. In this model the two particles with opposite spins are tied to an origin by a harmonic force field while they interact through a harmonic potential $u\left(r_{12}\right)=(1 / 2) \kappa r_{12}^{2}$, where, in an obvious notation, $r_{12}=\mid \mathbf{r}_{1}$ $-\mathbf{r}_{2} \mid$, rather than by $e^{2} / r_{12}$ to be treated later in this paper.

In units in which the interparticle spring constant $\kappa$ is measured relative to that of the external confining potential $\left(k_{\text {ext }}=1\right)$, the exact ground-state energy is known to be [6]

$$
E_{e x}=\frac{3}{2}\left[1+(1+2 \kappa)^{1 / 2}\right]
$$

while its Hartree-Fock counterpart is

$$
E_{H F}=3(1+\kappa)^{1 / 2} .
$$

Since the aim of this work is about correlation energy $\epsilon$, defined by

$$
\epsilon=E_{e x}-E_{H F},
$$

we note that it is readily demonstrated from Eqs. (2)-(4) that $\epsilon$ is of order $\kappa^{2}$ as $\kappa$ tends to zero.

Below, we shall proceed in the spirit of a Møller-Plessetlike perturbation theory (MPPT) by regarding $\epsilon$ as the (dimensionless, by suitable choice of units) expansion parameter to be employed. If we first focus on the diagonal element of the one-particle density matrix $\gamma\left(\mathbf{r}, \mathbf{r}^{\prime}\right)$, namely, the ground-state electron density $\rho(r) \equiv \gamma(\mathbf{r} ; \mathbf{r})$, from the above model it is known that

$$
\rho_{H F}(r)=\mathcal{N}_{H F} \exp \left[-(1+\kappa)^{1 / 2} r^{2}\right],
$$

whereas the exact ground-state density has a different exponent, but remains Gaussian in shape:

$$
\rho(r)=\mathcal{N} \exp \left[-\frac{(2 \alpha-1)}{\alpha} r^{2}\right],
$$

where

$$
\alpha=\frac{1}{2}\left[1+(1+2 \kappa)^{1 / 2}\right]
$$


with $\mathcal{N}_{H F}$ and $\mathcal{N}$ being normalization costants.

Here, because of the special forms of the two densities, it is possible to relate $\rho(r)$ and $\rho_{H F}(r)$ by the use of scaling, namely, by writing

$$
\rho(r)=\lambda^{3} \rho_{H F}(\lambda r) .
$$

Then $\lambda$ is readily obtained from Eqs. (5) and (6) as

$$
\lambda=\sqrt{\frac{2(1+2 \kappa)^{1 / 2}}{\left[1+(1+2 \kappa)^{1 / 2}\right](1+\kappa)^{1 / 2}}}=1+O\left(\kappa^{2}\right) .
$$

Such a result is clearly in the spirit of the MPPT, equations (8) and (9) can be expanded in an infinite series in the correlation energy $\epsilon, \rho_{H F}(r)$ being the leading term.

We proceed next to effect the off-diagonal generalization of Eq. (8), namely, to express $\gamma\left(\mathbf{r}, \mathbf{r}^{\prime}\right)$ in terms of its HF counterpart $\gamma_{H F}\left(\mathbf{r}, \mathbf{r}^{\prime}\right)$. The essential simplicity of twoelectron systems with opposed spins is that $\gamma_{H F}\left(\mathbf{r}, \mathbf{r}^{\prime}\right)$ can immediately be written in terms of the HF density as in Eq. (1). The objective in the remainder of the present study is to construct $\gamma\left(\mathbf{r}, \mathbf{r}^{\prime}\right)$, for the two-particle systems considered here, in terms solely of the exact density $\rho(r)$ and the HF density. Let us proceed to construct $\gamma\left(\mathbf{r}, \mathbf{r}^{\prime}\right)$ from the Moshinsky model. Following the study of March et al [7], from the definition of the appropriate wave function $\Psi\left(\mathbf{r}_{1}, \mathbf{r}_{2}\right)$, one can easily obtain

$$
\begin{aligned}
\gamma\left(\mathbf{r}, \mathbf{r}^{\prime}\right)= & 2\left(\frac{2 \alpha-1}{\pi \alpha}\right)^{3 / 2} \exp \left[-\frac{(2 \alpha-1)}{2 \alpha}\left(r^{2}+r^{\prime 2}\right)\right. \\
& \left.-\frac{(\alpha-1)^{2}}{4 \alpha} \mathbf{r}-\mathbf{r}^{\prime 2}\right] .
\end{aligned}
$$

Equation (10) can be expanded near the diagonal to give

$$
\gamma\left(\mathbf{r}, \mathbf{r}^{\prime}\right)=\rho(r)^{1 / 2} \rho\left(r^{\prime}\right)^{1 / 2}\left[1-\frac{(\alpha-1)^{2}}{4 \alpha} \mathbf{r}-\mathbf{r}^{\prime 2}\right],
$$

which is sufficient to get the exact kinetic-energy density by

$$
t(r)=\frac{1}{2}\left[\vec{\nabla}_{\mathbf{r}} \cdot \vec{\nabla}_{\mathbf{r}^{\prime}} \gamma\left(\mathbf{r}, \mathbf{r}^{\prime}\right)\right]_{\mathbf{r}^{\prime}=\mathbf{r}}
$$

In this exact model we can relate the kinetic energy to the total energy via the virial theorem and, because the same holds for the HF result, we can directly relate the correlation energy to the correlation kinetic energy. From the above definition (12), inserting $\gamma\left(\mathbf{r}, \mathbf{r}^{\prime}\right)$ from Eq. (11), the result is

$$
t(r)=\frac{|\vec{\nabla} \rho|^{2}}{8 \rho}+3 \rho(r) \frac{(\alpha-1)^{2}}{4 \alpha},
$$

which shows an "off-diagonal" contribution other than the so-called von Weizsäcker inhomogeneity kinetic-energy term.

Now, from the usual definition of the correlation kineticenergy density

$$
t_{\text {corr }}(r)=t(r)-t_{H F}(r)
$$

where $t_{H F}=\left|\vec{\nabla} \rho_{H F}\right|^{2} / 8 \rho_{H F}$, we readily obtain

$$
\begin{aligned}
t_{c o r r}(r)= & \rho(r)\left[\frac{(2 \alpha-1)^{2}}{2 \alpha^{2}} r^{2}+\frac{3(\alpha-1)^{2}}{4 \alpha}\right] \\
& -\rho_{H F}(r) \frac{(1+\kappa)}{2} r^{2}
\end{aligned}
$$

Thus, from the virial theorem

$$
\int t_{\text {corr }}(r) d \mathbf{r}=\frac{1}{2} \epsilon
$$

where $\epsilon$ is the correlation energy defined earlier. It is interesting to compare the diagonal (von Weizsäcker) and offdiagonal contributions to the correlation kinetic-energy. For the latter, $T_{o f f}$ say, we have immediately

$$
T_{o f f}=\frac{3(\alpha-1)^{2}}{2 \alpha},
$$

which behaves as $-\epsilon$ with very small deviations of order $0.075(-\epsilon)^{3 / 2}$ up to large values of $\kappa$. This is a remarkable result because we will show in the following section that, by changing the interparticle interaction to the Coulomb repulsion, the off-diagonal contribution to the correlation kineticenergy remains essentially of the same order $-\epsilon$. In the Moshinsky model, forced by the virial theorem, the diagonal contribution to the correlation kinetic-energy is thus of order $3 \epsilon / 2$.

Having obtained exact results for $\gamma\left(\mathbf{r}, \mathbf{r}^{\prime}\right)$ and $t_{\text {corr }}$ for the two-electron model atom, we start now to consider approximate relations for more realistic systems. We note first of all that, to obtain $t_{\text {corr }}$, we need only the near diagonal behavior of $\gamma\left(\mathbf{r}, \mathbf{r}^{\prime}\right)$. Therefore, we shall postulate the approximate form

$$
\begin{aligned}
\gamma\left(\mathbf{r}, \mathbf{r}^{\prime}\right)= & \frac{\rho(\mathbf{r})}{2}\left[\frac{\rho_{H F}\left(\mathbf{r}^{\prime}\right)}{\rho_{H F}(\mathbf{r})}\right]^{1 / 2}+\frac{\rho\left(\mathbf{r}^{\prime}\right)}{2}\left[\frac{\rho_{H F}(\mathbf{r})}{\rho_{H F}\left(\mathbf{r}^{\prime}\right)}\right]^{1 / 2} \\
& -f(\mathbf{r})^{1 / 2} f\left(\mathbf{r}^{\prime}\right)^{1 / 2}\left|\mathbf{r}-\mathbf{r}^{\prime}\right|^{2}+(\text { higher-order terms }),
\end{aligned}
$$

where $f(\mathbf{r})$ is a positive function directly related to the assumed Møller-Plesset-like expansion, and clearly is zero in the noninteracting limit. The rest of Eq. (18), apart from the near diagonal $\left|\mathbf{r}-\mathbf{r}^{\prime}\right|^{2}$ term, arises from the first-order Rayleigh-Schrödinger perturbation theory, as already noted in the study of Hall [8] (see also Ref. [9]), which generalized the density $\rho(\mathbf{r})$ given by Schwartz [10]. In the present model atom, it is straightforward to show from the exact $\gamma\left(\mathbf{r}, \mathbf{r}^{\prime}\right)$ in Eq. (10) that $f(\mathbf{r})$ is $\left[(\alpha-1)^{2} / 4 \alpha\right] \rho(r)$ and that the first term in Eq. (18) can be recovered by expanding the exact density around the HF counterpart and by truncating at the first order in the difference, namely, 


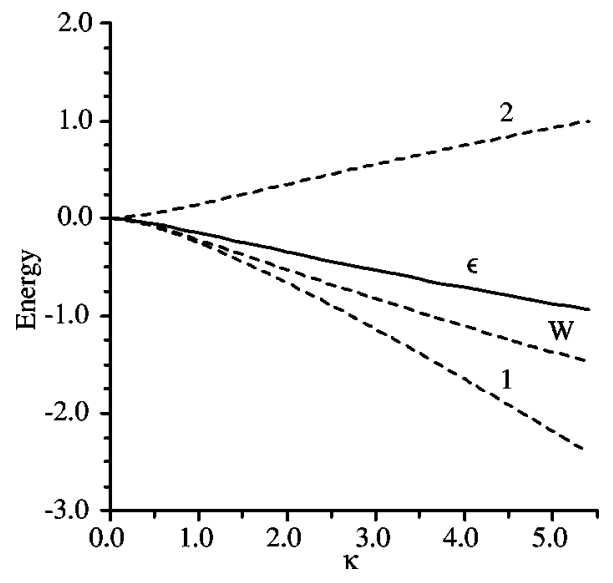

FIG. 1. Plot of correlation energy $(\epsilon)$ and correlation kineticenergy contributions (dashed lines) for the Moshinsky model atom against the interparticle spring constant $(\kappa)$. Data are in atomic units and the spring constant of the external confining potential is set to 1 . Curve $W$ refers to the von Weizsäcker contribution, curve 1 to the first two terms of Eq. (20) and curve 2 to the off-diagonal contribution (see text).

$$
\begin{aligned}
\rho(r)^{1 / 2} \rho\left(r^{\prime}\right)^{1 / 2}= & \rho_{H F}(r)^{1 / 2} \rho_{H F}\left(r^{\prime}\right)^{1 / 2}\left[1+\frac{\rho(r)-\rho_{H F}(r)}{\rho_{H F}(r)}\right]^{1 / 2} \\
& \times\left[1+\frac{\rho\left(r^{\prime}\right)-\rho_{H F}\left(r^{\prime}\right)}{\rho_{H F}\left(r^{\prime}\right)}\right]^{1 / 2} \\
= & \rho_{H F}(r)^{1 / 2} \rho_{H F}\left(r^{\prime}\right)^{1 / 2}\left[1+\frac{\rho(r)-\rho_{H F}(r)}{2 \rho_{H F}(r)}\right. \\
& \left.+\frac{\rho\left(r^{\prime}\right)-\rho_{H F}\left(r^{\prime}\right)}{2 \rho_{H F}\left(r^{\prime}\right)}+\cdots\right] .
\end{aligned}
$$

Returning to the kinetic-energy density, after a short calculation resulting from the insertion of Eq. (18) into Eq. (12), the approximate result for $t(\mathbf{r})$ is found to be

$$
t(\mathbf{r})=\frac{\vec{\nabla} \rho \cdot \vec{\nabla} \rho_{H F}}{4 \rho_{H F}}-\frac{\rho\left|\vec{\nabla} \rho_{H F}\right|^{2}}{8 \rho_{H F}^{2}}+3 f(\mathbf{r}) .
$$

In the Moshinsky model, the first two pieces on the righthand side of Eq. (20) give a contribution to the correlation kinetic-energy which behaves as $(3 / 2) \epsilon$ as $\kappa$ tends to zero, according to the von Weizsäcker term, but deviate significantly in the case of strong interparticle interaction $(\kappa>2)$. In Fig. 1 we show the plots of the contributions to the correlation kinetic-energy for the Moshinsky model atom against $\kappa$, according to the decompositions made in Eqs. (13) and (20).

\section{TWO-ELECTRON HOOKEAN ATOM}

This is the point at which to illustrate the physical basis for the approximation introduced into the density-matrix form (18). First we start from the Hookean atom in which two real electrons are confined by a harmonic potential with spring constant $k=1 / 4$ in atomic units. For such a system the exact ground-state wave function can be explicitly written [11],

$$
\Psi\left(\mathbf{r}_{1}, \mathbf{r}_{2}\right)=\mathcal{N}_{0}\left(1+\frac{1}{2} r_{12}\right) \exp \left[-\left(r_{1}^{2}+r_{2}^{2}\right) / 4\right]
$$

with energy eigenvalue $2 E_{h}$, and where the normalization constant $\mathcal{N}_{0}$ is given by

$$
\mathcal{N}_{0}^{2}=\frac{1}{4 \pi^{5 / 2}\left(8+5 \pi^{1 / 2}\right)} .
$$

The wave function (21) can be processed to give the exact one-electron density matrix. In particular, we are interested in determining a suitable function $f(r)$ in order to apply our proposal, Eq. (18), to this particular system. By writing

$$
\gamma\left(\mathbf{r}_{1}, \mathbf{r}_{2}\right)=2 \int \Psi\left(\mathbf{r}_{1}, \mathbf{r}_{3}\right) \Psi^{*}\left(\mathbf{r}_{2}, \mathbf{r}_{3}\right) d \mathbf{r}_{3},
$$

which is a function of $r_{1}, r_{2}$, and $r_{12}$, owing to spherical symmetry, we can define $f(r)$ by making the following limit:

$$
-f(r)=\lim _{\mathbf{r}_{1}, \mathbf{r}_{2} \rightarrow \mathbf{r}} \frac{1}{2}\left(\frac{\partial^{2}}{\partial r_{12}^{2}}\right) \underset{r_{1}, r_{2}}{ } \gamma\left(\mathbf{r}_{1}, \mathbf{r}_{2}\right),
$$

which leads to

$$
\begin{aligned}
-f(r)= & \lim _{\mathbf{r}_{1}, \mathbf{r}_{2} \rightarrow \mathbf{r}} \mathcal{N}_{0}^{2} \exp \left[-\left(r_{1}^{2}+r_{2}^{2}\right) / 4\right] \\
& \times\left(\frac{\partial^{2}}{\partial r_{12}^{2}}\right) \int_{r_{1}, r_{2}} \frac{1}{4} r_{13} r_{23} \exp \left(-r_{3}^{2} / 2\right) d \mathbf{r}_{3} .
\end{aligned}
$$

For the integration, it is convenient to choose a reference frame with $\mathbf{r}_{1}$ on the $x$ axis and $\mathbf{r}_{2}$ on the $x y$ plane. Developing the integrand up to the second order in $\theta_{12}$, the angle between the two vectors $\vec{r}_{1}$ and $\vec{r}_{2}$, one has

$$
f(r)=\frac{1}{4} \mathcal{N}_{0}^{2} \exp \left(-r^{2} / 2\right) \int \frac{y_{3}^{2} \exp \left(-r_{3}^{2} / 2\right)}{r^{2}+r_{3}^{2}-2 x_{3} r} d \mathbf{r}_{3} .
$$

For comparison purposes, a plot of this function $f(r) / f(0)$ and of the density ratio $\rho(r) / \rho(0)$ is shown in Fig. 2. For this particular case of the Hookean atom, $f$ is not proportional to the density as in the Moshinsky model, because by a fitting of data displayed in Fig. 2, we have obtained

$$
\frac{f(r)}{f(0)}=\left[\frac{\rho(r)}{\rho(0)}\right]^{a}
$$

with $a=1.867>1$. Integration of $3 f(r)$ gives finally $0.03383 E_{h}$, about $88 \%$ of $-\epsilon, \epsilon$, the correlation energy, being -0.03851 in this particular two-electron system. The HF solution is not analytically known; here it has been obtained by using a standard $a b$ initio program in which the 


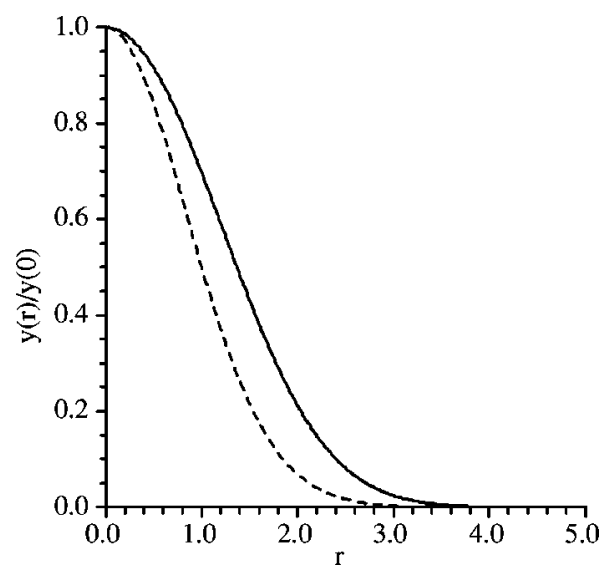

FIG. 2. Plot of $f(r) / f(0)$ from Eq. (26) (dashed line) and of the density ratio $\rho(r) / \rho(0)$ against $r\left(a_{0}\right)$ for the two electron Hookean atom considered in this work.

nuclear potential has been substituted by a harmonic potential. More precisely, $\rho_{H F}^{1 / 2}$, the doubly occupied $1 s$ orbital, has been constructed from $10 s$ Gaussian elementary functions with exponents $\alpha / 2^{n-1}$, with $\alpha=8$ and $1 \leqslant n \leqslant 10$. With the numerical $\rho_{H F}(r)$ and the analytical $\rho(r)$ we have also computed by numerical integration the correlation kinetic-energy contribution due to the two first terms on the right-hand side of Eq. (20). This contribution gives $0.00109 E_{h}$, very close to the von Weizsäcker contribution, namely, 0.00124 , and about $3 \%$ of $-\epsilon$. The rest of the correlation energy, less then $10 \%$, is recovered by the contribution due to the external harmonic potential. We remark, in fact, that in this model the virial theorem establishes that

$$
T=U_{e x t}-\frac{1}{2} U_{e e}
$$

where $T$ is the total kinetic-energy, $U_{e x t}$ is the potential energy of the electrons in the external harmonic force field, and $U_{e e}$ is the total electron-electron repulsion. Under these conditions the correlation energy $\epsilon$ can be written as

$$
\epsilon=-T_{\text {corr }}+3 U_{\text {ext }, \text { corr }} .
$$

In our numerical calculation, we obtained $U_{\text {ext,corr }}=$ $-0.00091 E_{h}$.

In the Hookean model, there are other analytical solutions for a discrete set of $k$ values smaller than 1/4 [12]. Such solutions correspond to a weaker confinement and consequently to stronger electron correlation effects. The application of the proposed equation (18) to the Hooke atom in this regime should lead to deviations similar to those observed in the Moshinsky model for high interparticle interaction but, in these cases, we have not attempted here any analytical development. The resulting $f(r)$ of Eq. (26) is strictly connected to the very simple form of the wave function for $k$ $=1 / 4$.

\section{HELIUMLIKE ATOMIC IONS}

Having discussed in some detail the proposal (18) for near diagonal $\gamma\left(\mathbf{r}, \mathbf{r}^{\prime}\right)$ of a two-electron system for Moshinsky and Hooke model atoms, we can now consider the realistic situation of two electrons in an external Coulomb potential due to a nuclear charge $Z e$. With standard ab initio programs it is possible to compute numerical HF and correlated densities. For this purpose we have considered the neutral $\mathrm{He}$ atom using a good enough Gaussian basis set made with $10 \mathrm{~s}$ functions, $5 p$ functions, and $3 d$ functions and a full configuration-interaction scheme. The resulting energies have been $-2.86110 E_{h}$ with $\mathrm{HF}$ and $-2.90071 E_{h}$ at a more refined level. In this framework the estimated value of the correlation energy is $-0.0396 E_{h}$, about $95 \%$ of the best literature data (see, for example, [13]). With the numerical $\rho(r)$ and $\rho_{H F}(r)$ we have finally computed the contribution to the correlation kinetic-energy from the first two terms of Eq. (20). This part of the correlation kinetic-energy contains the large $Z$ limit correlation kinetic-energy given earlier by Gál et al [14]. The result, $0.00083 E_{h}$, shows that in such systems the main contribution to the correlation kineticenergy comes again from the off-diagonal term $f(r)$. The virial theorem establishes now that $T_{c o r r}=-\epsilon$ and consequently

$$
3 \int f(r) d \mathbf{r} \simeq-\epsilon
$$

This result seems to be common in all cases in which the two electrons are trapped in a single-well potential. Equation (30) is essentially a consequence of the electron-electron cusp in the wave function, as it appears clearly in Eq. (25) that defines $f(r)$ for the Hookean atom with spring constant $1 / 4$. For the heliumlike atomic ions we can show, using simple arguments, that the correlation energy is weakly dependent on the nuclear potential. In fact, in terms of Koopmans theorem, one can write the total energy in the form

$$
E=-\frac{Z^{2}}{2}+\mu
$$

where $-Z^{2} / 2$ is the hydrogenic ion energy and $\mu$ is the HF eigenvalue for the two-electron system. The correlation energy is thus

$$
\epsilon=-\frac{Z^{2}}{2}+\mu-E_{H F}
$$

$E_{H F}$ being the HF total energy. By approximating the doubly occupied HF orbital with a $1 s$ hydrogenic orbital with exponent $Z-\sigma$, where $\sigma$ accounts for screening, one obtains

$$
\epsilon=-\frac{\sigma^{2}}{2}
$$

The value of $\sigma$ which minimizes the HF energy is 5/16 (see, for example, Ref. [15]) and leads to $\epsilon=-0.0488 E_{h}$, a slightly overestimated value. We remark that this value does not depend on $Z$ and could be used to estimate $f(r)$ by recognizing that, from the previous two models, 


$$
f(r) \leqslant \frac{f(0)}{\rho(0)} \rho(r)
$$

which leads to, combining Eqs. (30) and (33),

$$
f(r) \leqslant \rho(r) \frac{\sigma^{2}}{12}
$$

It is interesting to note that the exact correlation energy for the He-like ions ranges from $-0.042 E_{h}$ for $\mathrm{He}$ to $-0.047 E_{h}$ for large $Z[16]$.

\section{HYDROGEN MOLECULE}

In the hydrogen molecule, the two electrons are placed in a double-well potential. At the dissociation, when the two nuclei are far apart, there is not any contribution to the correlation energy due to the electron-electron cusp, because the electrons themselves are far apart on each nucleus. Nevertheless the correlation energy is much bigger in modulus than in the $\mathrm{He}$ atom. This effect is due to the fact that in $\mathrm{HF}$ at dissociation there is a residual mean-field electron-electron interaction.

At infinite interatomic distance we still have from the virial theorem that $T_{\text {corr }}=-\epsilon$ because the derivative of the energy with respect to the internuclear distance is zero. Thus we can compare again the correlation kinetic-energy with $\epsilon$. For the exact density, we can write

$$
\rho(\mathbf{r}) \simeq \frac{1}{\pi}\left[e^{-2\left|\mathbf{r}-\mathbf{R}_{1}\right|}+e^{-2\left|\mathbf{r}-\mathbf{R}_{2}\right|}\right] \quad\left[\left|\mathbf{R}_{1}-\mathbf{R}_{2}\right| \rightarrow \infty\right],
$$

where $\mathbf{R}_{1}$ and $\mathbf{R}_{2}$ indicate the positions of the two protons, while for the HF density we have

$$
\rho_{H F}(\mathbf{r}) \simeq \mathcal{N}\left[\phi\left(\left|\mathbf{r}-\mathbf{R}_{1}\right|\right)^{2}+\phi\left(\left|\mathbf{r}-\mathbf{R}_{2}\right|\right)^{2}\right],
$$

where $\phi$ is a spherical symmetry orbital to be determined by minimizing the functional

$$
F=\left\langle\phi\left|-\frac{1}{2} \nabla^{2}-\frac{1}{r}\right| \phi\right\rangle+\frac{1}{4} \iint \frac{\phi\left(r_{1}\right) \phi\left(r_{2}\right)}{r_{12}} d \mathbf{r}_{1} d \mathbf{r}_{2},
$$

where the repulsion integral corresponds to the residual electron-electron mean-field interaction. The optimal $F$ is one-half of the HF total energy at dissociation. In this case, again, a combination of ten Gaussian elementary functions is sufficient for a good approximation of $\phi$. In our calculation we have obtained $-0.71516 E_{h}$ for the HF energy and consequently $-0.28484 E_{h}$ for $\epsilon$. Finally, neglecting the term in $f$, we have evaluated the correlation kinetic-energy from Eq. (20): the result $0.26938 E_{h}$ is in substantial agreement with the expected value and shows that the proposed equation (18) for the one-particle density matrix takes into account both short- and long-range correlations in the ground-state realistic two-electron systems.

\section{SUMMARY AND FUTURE PERSPECTIVES}

The main achievements of the present study are the following: (i) the summation to all orders of a Møller-Plessetlike series, relating the exact density $\rho(r)$ to the HF density $\rho_{H F}(r)$, embodied in Eq. (8) for the "model atom" of Moshinsky [6]; (ii) its generalization to the one-particle density matrix $\gamma\left(\mathbf{r}, \mathbf{r}^{\prime}\right)$ and the exact kinetic-energy density $t(r)$ for the same model; (iii) the approximate proposal (18) for the near-diagonal $\gamma\left(\mathbf{r}, \mathbf{r}^{\prime}\right)$, which can be used to construct the kinetic-energy density in all realistic two-electron systems. The proposed expression for the 1DM depends solely on the (assumed known) ground-state density, the corresponding HF density and a positive function $f(\mathbf{r})$ which can be related to the density itself and does not contribute to the diagonal. It has been proved that this function $f(\mathbf{r})$ is essentially related to the short-range interparticle correlation; in the case of electrons, which show Coulomb repulsion, trapped in a harmonic force field with spring constant 1/4, $f(\mathbf{r})$ can be evaluated analytically and it is shown here that it is related to the electron-electron cusp in the wave function. The other term of $\gamma\left(\mathbf{r}, \mathbf{r}^{\prime}\right)$ in Eq. (18) which gives the exact density on the diagonal is able instead to account for long range correlation, as is shown for the hydrogen molecule at dissociation. The expression (18) behaves properly for all types of force field and seems to be valid up to relatively intense interparticle interactions. The computed kineticenergy, for the different cases treated in this work, is in general agreement with the virial theorem-a result we think promising for future applications. Moreover, if one contemplates approximations for $f(\mathbf{r})$ as that of Eq. (35), the kinetic-energy density $t(\mathbf{r})$ resulting from Eq. (20) is linear in the exact density - a remarkable aspect in density functional theory.

An interesting suggestion for the future consists in an extension of the present study to more complicated wave functions written in terms of two-particle group functions (geminals) $[17,18]$. In this area, very recently Nagy [19] has shown that the problem of an arbitrary system with even electrons can be reduced to a two-particle problem and that the effective potential in the two-electron equation contains a term of completely kinetic origin.

\section{ACKNOWLEDGMENTS}

C.A. acknowledges financial support from MURST (Progetti di interesse nazionale). N.H.M. wishes to thank Scuola Normale Superiore and in particular Professor M.P. Tosi for generous hospitality during his visit to Pisa in 2002, where some of this work was carried out. N.H.M. also acknowledges partial financial support from the Franqui Foundation (Brussels) and wishes to thank especially Professor L. Eyckmans of that Foundation for encouragement and motivation. Finally the authors thank Professor I.A. Howard, Professor Á. Nagy, Dr. F. Bartha, Dr. F. Bogar, and Dr. T. Gál for valuable discussions in this general area. 
[1] A. Holas and N.H. March, Phys. Rev. A 51, 2040 (1995).

[2] R.G. Parr and W. Yang, Density Functional Theory of Atoms and Molecules (Oxford University Press, Oxford, 1989).

[3] M. Levy and N.H. March, Phys. Rev. A 55, 1885 (1997).

[4] S. Goedecker and C.J. Umrigar, Phys. Rev. Lett. 81, 866 (1998).

[5] A. Holas, Phys. Rev. A 59, 3454 (1999).

[6] M. Moshinsky, Am. J. Phys. 36, 52 (1968).

[7] N.H. March, I.A. Howard, I. Nagy, and A. Rubio, Phys. Lett. A 288, 101 (2001).

[8] G.G. Hall, Adv. Quantum Chem. 1, 241 (1964).

[9] C. Amovilli, N.H. March, T. Gál, and Á. Nagy, Int. J. Quantum Chem. 77, 716 (2000).
[10] C. Schwartz, Ann. Phys. (N.Y.) 6, 156 (1959).

[11] S. Kais, D.R. Herschbach, and R.D. Levine, J. Chem. Phys. 91, 7791 (1989).

[12] M. Taut, Phys. Rev. A 48, 3561 (1993).

[13] V.I. Korobov, Phys. Rev. A 61, 064503 (2000).

[14] T. Gál, N.H. March, and Á. Nagy, Chem. Phys. Lett. 305, 429 (1999).

[15] L.D. Landau and E.N. Lifshitz, Quantum Mechanics: Nonrelativistic Theory (Pergamon Press, London, 1958).

[16] E. Clementi, J. Chem. Phys. 38, 2248 (1963).

[17] A.J. Coleman, Int. J. Quantum Chem. 63, 23 (1997).

[18] D. Mazziotti, J. Chem. Phys. 112, 10125 (2000).

[19] Á. Nagy, Phys. Rev. A 66, 022505 (2002). 